\title{
Antioxidants Properties of Natural and Synthetic Chemical Compounds: Therapeutic Effects on Biological System
}

\author{
Chika J Mbah ${ }^{1}$, Ifeoma Orabueze ${ }^{2}$ and Ndiamaka H Okorie ${ }^{3 *}$ \\ ${ }^{1}$ University of Nigeria, Nsukka, Nigeria \\ ${ }^{2}$ University of Lagos, Nigeria \\ ${ }^{3}$ Enugu State University of Science and Technology, Nigeria \\ *Corresponding Author: Ndiamaka H Okorie, Enugu State University of Science and Technology, Nigeria.
}

Received: March 18, 2019; Published: May 03, 2019

DOI: $10.31080 /$ ASPS.2019.03.0273

\begin{abstract}
The imbalance in homeostatic process between oxidants and antioxidants in the body caused by reactive oxygen and nitrogen species (free radicals) leads to oxidative stress. Cellular enzymes such as myeloperoxidase, nicotinamide adenine dinucleotide phosphate-oxidase (NADPH- oxidase) and nitric oxide synthase (NOS) produce these reactive oxygen and nitrogen species (RONS). Oxidative stress is considered to be the primary cause of aging and a wide variety of human diseases such as cancers, diabetes, neurodegenerative disorders (Alzheimer's disease, Parkinson's disease), rheumatoid arthritis etc. Invariably, to maintain a healthy biological system the balance between oxidation and antioxidation is a critical requirement. Antioxidants are any substances that significantly delay, prevent or inhibit oxidative damage to target molecules. They are widely distributed throughout plants and animals and are found to be at low concentrations compared with that of oxidizable substrates. Natural and synthetic antioxidants have been reported to prevent or treat various human diseases. This article presents a comprehensive account of free radical generation, oxidative stress and its consequences, antioxidants including sources, classification, chemical structures, mechanisms of action, physicochemical properties. The study also dealt with applications of antioxidants in pharmaceutical and food industries, their therapeutic properties and pharmacokinetic features.
\end{abstract}

Keywords: Free Radicals; Oxidative Stress; Antioxidants; Therapeutic Properties; Pharmacokinetics

\section{Introduction}

Oxidation is a chemical reaction that involves the transfer of electrons from one compound to another and has negative effects physiologically. Biologically, oxidation is often started by free radicals. An antioxidant is a substance (at low concentration) when compared to a substrate to be oxidized, significantly delays or inhibits the oxidation of that substrate. The physiological role of antioxidant, therefore is to prevent damage to cellular components resulting from chemical reactions that involve free radicals. A free radical is a highly reactive chemical species that contains one or more unpaired electrons [1]. Free radicals have played vital roles in many fundamental cellular reactions and are implicated in etiology of various human disorders. In humans for instance, damage by free radicals is counteracted with physiological (natural) antioxidants namely catalase, glutathione, glutathione peroxidases, superoxide dismutases, ubiquinol, uric acid, essential minerals and nutritional antioxidants from diet such as vitamins $\mathrm{E}$, vitamin $\mathrm{C}$, carotenoids. The objective of the present review is to consider a number of properties of antioxidants such as free radical generation, oxidative stress and its consequences, sources and classifications, chemical structures and mechanism of actions. Their therapeutic and pharmacokinetic properties were also considered. Literature search has revealed that numerous articles have been reported on antioxidants in under various topics however, little or no information has been reported on the topic of the present review. 
Formation of hydroxyl radical from hydrogen peroxide

$$
\text { c. } . \mathrm{OH}+\mathrm{RH} \quad \mathrm{O}_{2}, \mathrm{ROO}+\mathrm{H}+-------- \text { Eq. } 13 .
$$

Formation of peroxyl free radical by direct reaction with organic substrate

$$
\text { d. } \quad \mathrm{OH}+\mathrm{LH} \quad \mathrm{O}_{2}, \quad \mathrm{LOO}+\mathrm{H}^{+}--\cdot------ \text { Eq. } 14 .
$$

Formations of peroxyl free radical by direct reaction with lipid

\section{Biological role of free radicals}

The beneficial effects of reactive species (ROS and RNS) occur at low/moderate concentrations but may be harmful as the accumulation of the reactive species increased [5]. Most cells can produce superoxide, hydrogen peroxide and nitric oxide constitutively while others have inducible ROS/RNS release system. Some of the major beneficial activities of ROS and RNS at low concentrations are (i) detoxification of xenobiotics by Cytochrome P450; (ii) defense against infectious agents by phagocytosis; (iii) generation of ATP in mitochondria (energy production); (iv) killing of cancer cells by macrophages and cytotoxic lymphocytes; (v) cell growth, and (vi) the induction of mitogenic responses [6]. Also at low concentrations, they exhibit vital roles on different cellular signals such as activation of nuclear transcription factors, activation of several cytokines and growth factor signals, non-receptor tyrosine kinases activation, activation of protein tyrosine phosphatases, release of calcium from intracellular stores, gene transcription and regulation of soluble guanylate cyclase activity in cells [7]. Nitric oxide (NO) generated by endothelial cells is essential for angiogenesis, blood pressure regulation of vascular smooth muscle, leukocyte adhesion, platelet aggregation and thrombosis while those produced by neurons are important neurotransmitters and key for neural plasticity. Furthermore, nitric oxide generated by activated macrophages is a principal mediator of the immune response.

\section{Oxidative stress}

Cells normally have a number of mechanisms to defend against damage induced by these reactive species. However, when the production of RONS exceeds the ability of cells to defend against these reactive substances, an imbalance occurs between the systemic manifestation of free radicals and the capability of cells to detoxify them [8]. Such an imbalance is called oxidative stress. The damaging effects of oxidative stress involve cellular damage and subsequent cell death because the reactive species oxidize critical cellular components, such as lipids, proteins, and DNA.

\section{Sources of Oxidative Stress}

Oxidative stress can be broadly divided into exogenous and endogenous types. Exogenous sources of oxidative stress are (i) ionizing radiation such as alpha $(\alpha)$, beta $(\beta)$, gamma $(\gamma)$ particles respectively from radioactive decay as well as X-ray photons [9]; (ii) pesticides, environmental chemicals and pollutants [10]; (iii) redox-active metals such as iron (Fe), copper $(\mathrm{Cu})$, chromium (Cr), and cobalt (Co)) [11]. Endogenous source of stress is mainly derived from signaling pathways, metabolic and/ or inflammation processes and often has an intracellular origin [12]. Metabolic processes may produce different types of reactive species and may involve enzymatic and non-enzymatic processes. Enzymatic processes are cytochrome P450 enzymes, lipoxygenase, cyclooxygenase, NADPH-oxidase, xanthine oxidase and uncoupled endothelial nitric oxide synthase (eNOS) while non-enzymatic processes producing reactive species by mitochondrial respiratory chain is complex because reactive species can be beneficial from metabolic standpoint however, detrimental to cells [13].

\section{Oxidative stress and human diseases}

An important relationship has been observed between oxidative stress and a wide variety of human diseases. Such diseases include:

- Cardiovascular disease: Reactive oxygen species-induced oxidative stress plays a key role in the pathogenesis of different cardiovascular diseases such as atherosclerosis, ischemic heart disease, hypertension, cardiomyopathy, cardiac hypertrophy and congestive heart failure. Induction of $\mathrm{Ca}^{2+}$ ion overload by these reactive oxygen species (ROS) leads to changes in membrane permeability, membrane lipid bilayer disruption, functional modification of various cellular proteins; abnormalities in myocyte function and endothelial dysfunction [14].

- Diabetes: Oxidative stress in normal cell contribute to both type- 1 (insulin dependent) and type-2 (non-insulindependent) diabetes mellitus. Stimulation of reactive species could come from cytochromeP450 monooxygenases, glucose auto-oxidation, NADPH-oxidase, lipooxygenase, nitric oxide synthase and oxidative phosphorylation [15]. 
- $\quad$ Neurodegenerative diseases: Oxidative stress arising from ROS generated by factors like exposure to herbicides, industrial chemicals, trace metals, cyanide, organic solvents, carbon monoxide and carbon disulphide may increase the risk of developing neurodegenerative disorders in particular Parkinson's disease [16]. The diseases are characterized by loss of nerve cells from brain and spinal cord, leading to either functional loss (ataxia) or sensory dysfunction (dementia), mitochondrial dysfunctions and apoptosis [17]. Examples of such disorders are Parkinson's disease, Alzheimer's disease, multiple sclerosis, amyolotrophic lateral sclerosis, Huntington's disease, cognitive dysfunction in the elderly, schizophrenia and tardive dyskinesia.

- Cancer: Deoxyribonucleic acid (DNA) damage and mutation play critical roles in carcinogenesis [18]. Redox potentials of reactive species (RNS, ROS) may play important roles in sequence-specific DNA damage arising from activation of activator protein (AP1) and nuclear factor kappa B (NF- $\kappa \mathrm{B})$ signal transduction pathways leading to the transcription of genes involved in cell growth regulatory pathways and pathogenesis of cancer.

- Rheumatoid arthritis: Is a chronic inflammatory autoimmune disease characterized by erosive, progressive and chronic polyarthritis. The pathogenesis of rheumatoid arthritis arises from the formation of free radicals at the site of inflammation leading to lipid peroxidation modification of low density lipoprotein, inactivation of alpha-1-protease inhibitor, DNA damage, activation of neutorphil, NADPH oxidase and endothelial cell xanthine dehydrogenase [19] Decrease in the concentrations of whole blood glutathione and total thiols also contribute to rheumatoid arthritis..

- Gastrointestinal disorder: Oxidative stress has been found as a causative factor for almost all gastrointestinal diseases. This arises probably from the enzymatic processes in gastrointestinal tract (GIT) necessary to form large amounts of oxygen radicals [20].

- Renal system disorder: Renal system is susceptible to reactive oxygen species (ROS) induced damage. These reactive species are generated by glomerular (endothelial), vascular (endothelial and smooth muscle cells) and tubular (proximal, distal and collector) cells of renal structure by stimulating potential factors [21].
- Pulmonary disorder: Inflammations of airways arising from oxidative stress is responsible for the pathogenesis of chronic pulmonary disorders such as asthma and chronic obstructive pulmonary disease (COPD) [22]. The risk of pulmonary disorders is further amplified by free radicals generated by ozone, cigarette smoking (air pollutants), infections, and other allergens. These reactive oxygen species act by causing direct oxidative damage to epithelial cells of the airways or may evoke bronchial hyper-reactivity and stimulation of histamine release from mast cells of the airways epithelial cells.

- $\quad$ Eye disorder: The eye is one of the most susceptible organs to oxidative damage caused by atmospheric oxygen, abrasion, light and toxins. Ultraviolet light also increases generation of reactive oxygen species such hydrogen peroxide, superoxide and hydroxyl radicals. Oxidative stress caused by these ROS is responsible for eye disorders such as cataracts, glaucoma and macular degeneration [23]. Conversion of light energy into a nerve impulse by the photoreceptors could be the mechanism of action.

- Infertility and pregnancy: Oxidative stress has been implicated in male and female infertility [24]. Reactive oxygen species (ROS) can exhibit beneficial or detrimental effects on reproductive system depending on the location, concentration, as well as the length of exposure to these reactive species. Reactive oxygen species are being generated from spermatozoa, leucocytes and an increased concentration levels in semen correlate negatively with sperm concentration, motility, fusion events associated with fertilization and subsequently leads to infertility in male [25]. Oxidative stress also influences multiple physiological processes such as oocyte maturation, fertilization, implantation, embryo development and therefore has been implicated in endometriosis, polycystic ovarian disease, unexplained infertility and recurrent pregnancy loss.

- Aging: Aging is defined as a progressive decline in the physiological functions of an organism after the reproductive phase of life. Oxidative stress is responsible for damage of different cell components and is the key components of age related disorders. Among the genetic theory and damageaccumulation theories used to explain the aging process, free radical theory is probably the most complex approach. It is based on the fact that the random deleterious effects of free radicals produced during aerobic metabolism cause damage to DNA, lipids and proteins [26]. 


\section{Antioxidants}

To enhance the ability of cells to defend against these reactive species (free radicals), certain natural and chemical compounds called antioxidants have the properties to perform such function.

The capability of cells to detoxify these reactive species can be illustrated below.

$$
\mathrm{O}_{2}^{-} \stackrel{\text { SOD }}{\longrightarrow} \mathrm{H}_{2} \mathrm{O}_{2}+\mathrm{O}_{2}-------- \text { Eq. } 15 \text {. }
$$

The equation 15 shows how superoxide radical $\left(\mathrm{O}_{2}^{-}\right)$is detoxified by the superoxide dismutase (SOD) enzyme to form hydrogen peroxide $\left(\mathrm{H}_{2} \mathrm{O}_{2}\right)$ and oxygen $\left(\mathrm{O}_{2}\right)$.

$$
\mathrm{H}_{2} \mathrm{O}_{2} \stackrel{\mathrm{GSHP}_{\mathrm{X}}}{\longrightarrow} \mathrm{H}_{2} \mathrm{O}+\mathrm{O}_{2}-\cdots-\cdots \text { Eq. } 16 .
$$

The equation 16 shows how hydrogen peroxide $\left(\mathrm{H}_{2} \mathrm{O}_{2}\right)$ is converted by the glutathione peroxidase (GSH-Px) enzyme to form water and oxygen, therefore preventing the accumulation of a precursor to free-radical biosynthesis.

Antioxidant as relates to the biological system is any substance that when present at low concentrations compared to those of an oxidizable substrate significantly delays, prevents or inhibit oxidation of that substrate [27]. Such substrates include carbohydrates, fats and oil (lipids), proteins and deoxyribonucleic acid (DNA). Biological antioxidants also include repair systems such as antioxidant enzymes, iron transport proteins (e.g. transferrin, albumin, ferritin and caeruloplasmin) and factors affecting vascular homeostasis [28]. However, as it relates to food, antioxidant is a substance that in small quantities is capable of preventing, delaying or greatly retarding the oxidation of easily oxidizable materials leading to the development of rancidity or other flavour deterioration [29]. In a nutshell, the role of an antioxidant is to intercept a free radical before it can react with the substrate. Therefore, antioxidants of biological/therapeutic importance should have the ability to react/ trap the free-radical before it reacts with the susceptible substrate and initiate chain reaction.

\section{Mechanisms of antioxidants}

Antioxidants may exert their effects by (i) inducing the biosynthesis of other antioxidants or defense enzymes (ii) suppressing the formation of active species (iii) reducing hydroperoxides (ROO) and $\mathrm{H}_{2} \mathrm{O}_{2}$ (iv) sequestering metal ions (v) scavenging active free radicals (vi) repairing and/or cleaning damage [30]. These mechanisms are illustrated below.

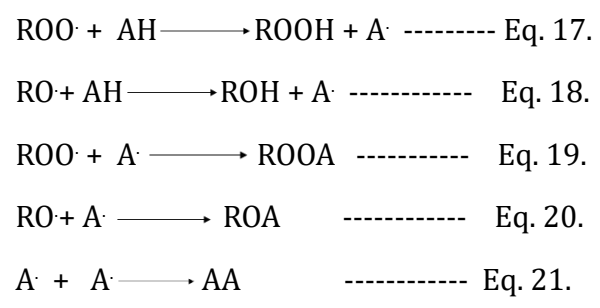

Equations 17 and 18 represent antioxidants donating hydrogen atoms to free radicals and thus terminating their lives. The antioxidant molecule is represented by $\mathrm{AH}$, in which ' $\mathrm{H}$ ' is the active hydrogen and the free radicals are represented by lipid radicals. The products are lipid derivatives and antioxidant radicals that are more stable.

Equations 19 and 20 represent antioxidant radicals participating in termination reactions with peroxy and oxy free radicals.

Equation 21 represents formation of antioxidant dimmers (dimerization) This effectively ends the autocatalytic free radical chain mechanism provided the antioxidant is present in its nonradical form.

\section{Characteristics of antioxidants}

Monohydroxy or polyhydroxy phenol compounds with various ring substitutions are the most current antioxidants utilized in foods. As they have low activation energy, the compounds can easily donate hydrogen and the resulting antioxidants radical unable to initiate another free radical due to the stabilization of the delocalized radical electrons. The donation of hydrogen from the antioxidants, delay or minimize the initiation and propagation of free radicals chain reaction. The resulting antioxidant free-radical is not subject to rapid oxidation due to its stability and can react with lipid free radicals to form a stable complex compound thus preventing them from being damaged.

\section{Classification of antioxidants}

Antioxidants are categorized in two major groups namely natural and synthetic [31]. These groups are in dietary antioxidants intakes and play major roles in maintaining the homeostasis of the oxidative balance. They are believed to protect humans from disease and aging. Sub-classification based on solubility contains oil-soluble antioxidants and water soluble antioxidants while those based on the mechanisms of action are primary antioxidants (radical scavengers), secondary antioxidants (peroxide decomposers), and metal deactivators. 


\section{Natural (primary) antioxidants}

Natural antioxidants are compounds found in foods consumed without much processing such as fruits, vegetables, nuts, grains, seeds, animal tissues etc. They are the chain breaking chemical compounds that react with lipid radicals to convert them into more stable products [32] Natural antioxidants can be extracted from plants, microorganisms and animal tissues. They may possess several drawbacks including high usage levels, low antioxidant efficiency, undesirable flavour or odour, and possible loss during processing. These drawbacks occasionally call for their replacement by synthetic chemicals, which are cheaper, more easily available, of consistent quality, and have greater antioxidant activity. Other sources of these antioxidants in plants are cereals, legumes, tea, coffee, wine, beer, herbs and spices (thyme, nutmeg, clove, black pepper, ginger, garlic, curcumin and derivatives) $[33,34]$. The animal sources include milk, fish lipids, eggs, glutathione (non-protein in animal tissue), carotenes and ubiquinone (coenzyme Q).

\section{Examples of natural antioxidants}

Minerals - are co-factors of antioxidants enzymes. Their absence affect metabolism of many macromolecules such as carbohydrates, proteins and lipids. Examples include copper, iron, manganese, selenium and zinc.

\section{Flavonoids}

- Catechins (roanthocyanidins) - are flavanols, or flavan-3-ols. Major catechins are catechin, epicatechin, epicatechin gallate (ECG), and epigallocatechin-3-gallate (EGCG) that inhibit lipid oxidation in red meat, poultry and fish.

- Carotenoids: Carotenoids are a group of isoprenoids synthesized by plants and microorganisms. Most of them have long carbon chain skeleton with series of conjugated carbon double bonds. Typical examples are lycopene, lutein, $\alpha$-carotene and $\beta$-carotene.

- Anthocyanins - are natural colorants/pigments of fruits and vegetables. They are novel antioxidants and potent inhibitors of lipid peroxidation as compared to other classic antioxidants.The positively charged oxygen atom in the anthocyanin molecule makes it a more potent and distinct hydrogen donating antioxidant compared to oligomeric proanthocyanidins (OPCs) and other flavonoids.

Honey - a natural product formed from nectar by honeybees and has antioxidant properties. These properties are due to the presence of phenolic and flavonoid compounds such as benzoic acid, cinnamic acid, chlorogenic acid, caffeic acid, ferulic acids, ellagic acid, gallic acid, syringic acid, myricetin, hesperetin, coumaric acid, isoramnetin, chrysin, quercetin, galangin, luteolin, kaempferol and isoramnetin,. In addition, it contains ascorbic acid, other organic acids and trace elements such as selenium.

Vitamins

- Retinol (Vitamin A) - Vitamin A is obtained from the diet either as preformed vitamin A or as provitamin A carotenoids. Preformed vitamin A is ingested as long-chained fatty acids of retinol in foods such as butter, eggs, milk, liver and fortified cereals. Provitamin A carotenoids ( $\alpha$-carotene, $\beta$-carotene, and -cryptoxanthin) are found in vegetables such as carrots, spinach, collards, pumpkins, and squash.

- $\quad$ Ascorbic acid (vitamin C) - widespread in plant tissues It is gamma lactone of simple sugar. The antioxidant activity of ascorbic acid depends on its capacity to bind metal ions.

- Tocopherols (vitamin E) - widely distributed in plant tissues, vegetables, animal fats, wheat germ oil and cottonseed oil. Antioxidant activity depends on concentration and temperature.

- Phospholipids - Lecithin and cephalin are important commercial antioxidants.

Table 1 provides summary of the natural antioxidants and their sources.

\begin{tabular}{|c|c|}
\hline Antioxidants & Sources \\
\hline $\begin{array}{l}\text { Ascorbic acid } \\
\text { Catechins } \\
\text { Anthocyanins } \\
\text { Beta-carotene } \\
\text { Polyphenols } \\
\text { Lycopene } \\
\text { Tocopherol } \\
\text { Lutein } \\
\text { Selenium }\end{array}$ & $\begin{array}{l}\text { Citrus fruits, tomatoesvegetables, brown rice, } \\
\text { pink and red wine, mango, papaya, sweet } \\
\text { potato, watermelon. } \\
\text { Apples, beans, berries, wine, tea, fruits, } \\
\text { chocolate. } \\
\text { Beets, berries, egg plant, grape fruits, } \\
\text { pink and red wine. } \\
\text { Vegetables, tomatoes, carrots, sweet potatoes, } \\
\text { apricots, papayas, squash, acorn, peaches, bell } \\
\text { peppers, broccoli, mango. } \\
\text { Tea, fruits, vegetables, red cabbage, blue and } \\
\text { black berries. } \\
\text { Tomatoes, papaya, watermelon, guava, pink } \\
\text { and red grapes. } \\
\text { Cereals, broccoli, brussels sprouts, beans, } \\
\text { cauliflowers, } \\
\text { cooking oils (olive, sunflower, safflower), } \\
\text { almonds, } \\
\text { hazenuts, whole grains, wheat germ. } \\
\text { Corn, egg. } \\
\text { Carrots, chicken, garlic, onions, oat meal, } \\
\text { salmon, tuna, seafood, whole grains, wheat } \\
\text { germ. }\end{array}$ \\
\hline
\end{tabular}

Table 1: Natural antioxidants and their sources [33,34]. 
Chemical structures and physicochemical properties of some natural antioxidants

\section{Retinol (Vitamin A)}

Chemical structure<smiles>CC(/C=C/C=C(C)/C=C/C=C(C)/C=C/CO)=C1CCCC1(C)C</smiles>

Figure 1: Vitamin A (natural water insoluble vitamin).

Physicochemical properties

A white crystalline powder. Practically insoluble in water or glycerol. Soluble in absolute ethanol, methanol, chloroform, ether, fats and oils. It has a melting point of $137-138^{\circ} \mathrm{C}$.

\section{Ascorbic acid (Vitamin C)}

Chemical structure

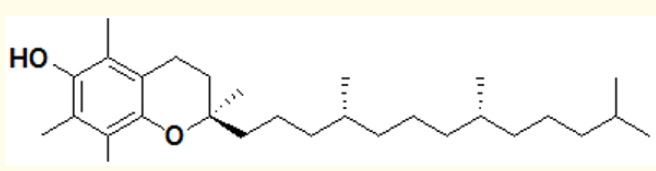

Figure 2: Vitamin C (natural water soluble vitamin).

Physicochemical properties

Vitamin $\mathrm{C}$ is $\gamma$-lactone of a $\mathrm{C}_{6}$ acid (hexose) having double bond between $\mathrm{C}_{2}$ and $\mathrm{C}_{3}$. The naturally occurring form (L-ascorbic acid) is a white, crystalline powder, odorless. It is a highly water soluble substance. The stability is lost by heat and aerobic oxidation, particularly in alkaline solutions and cooking. It is a powerful reducing agent that is stable below pH 6.8 at room temperature. Oxidation of ascorbic acid yields dehydroascorbic acid.

\section{Vitamin E}

Chemically defined as (2R)-2,5,7,8-Tetramethyl-2-[(4r,8r)4,8,12-trimethyltridecyl]-3,4-dihydrochromen-6-ol.

Chemical structure

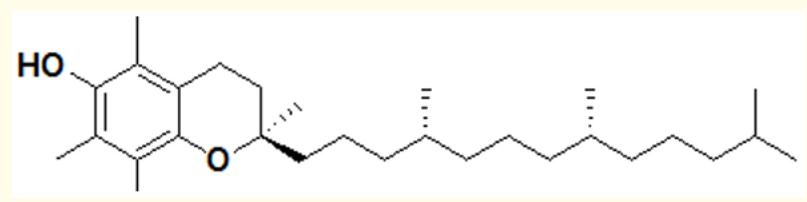

Figure 3: Vitamin E (natural water insoluble vitamin).
Physicochemical properties

It is aclear viscous oil (slightly pale liquid) that is odorless and darkens upon exposure to air. It is insoluble in water, miscible with ether and freely soluble in ethanol. The density is $0.950 \mathrm{~g} / \mathrm{cm}^{3}$ while the refractive index [n]D/20 is 1,503-1,507.

\section{Synthetic (secondary) antioxidants}

Synthetic antioxidants are phenolic compounds that perform the function of capturing free radicals and stopping the chain reactions. They are compounds produced artificially and added to processed or pre-packaged food to prevent rancidity, browning. The most used synthetic antioxidants include butylated hydroxy anisole (BHA), butylated hydroxy toluene (BHT), tertiary-butylhydroquinone (TBHQ), nordihydro guaretic acid (NDGA), propyl gallate (PG), octyl gallate, dodecyl gallate and metal chelating agents (ethylene diamine tetra acetic acid, polyphosphatases).

These synthetic antioxidants are easier to use, more reliable, and generally more cost-effective than natural antioxidants. They are also expected to be non-toxic, highly active at low concentrations $(0.01-0.02 \%)$, concentrate on the surface of the fat or oil phase, relatively stable and able to penetrate the cells [35]. Used in vegetable oils, baked and confectionary products, cosmetics and margarine.

Chemical structures and physicochemical properties of synthetic antioxidants

Butylated hydroxy anisole (BHA)

Consists of, a mixture of 3-tert-butyl 4-methoxyphenol and 2 tert butyl-4 methoxyphenol.

Chemical structure<smiles>COc1ccc(O)cc1C(C)(C)C</smiles>

Figure 4: Butlated hydroxyl anisole (synthetic water insoluble antioxidant).

Physicochemical properties

White waxy flakes, soluble in fats, oils and most organic solvents but insoluble in water. The stability of fat containing BHA decreases when they are heated to high temperature. Melting point is $50-52^{\circ} \mathrm{C}$ [36]. 
Butylated hydroxy toluene (BHT)

Chemically defined as 2,6-di-tert butyl-4-methylphenol.

Chemical structure

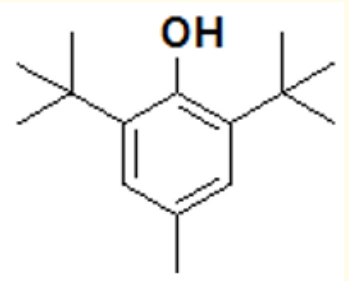

Figure 5: Butylated hydroxyl toluene (synthetic water insoluble antioxidant).

Physicochemical properties

It a is white crystalline solid. The compound is insoluble in water but soluble in fats, oils and most organic solvents. Melting point is $68-70^{\circ} \mathrm{C}$

Tertiary-butylhydroquinone (TBHQ)

Chemical structure<smiles>CC(C)(C)c1cc(O)ccc1O</smiles>

Figure 6: Tertiary-butylhydroquinone (synthetic water insoluble antioxidant).

Physicochemical properties

It is a beige or white-to-tan crystalline powder. Solubility decreases in order of alcohol $>$ fats $>$ water. It is stable to heat and is most effective antioxidant in preventing oxidation of frying oils. Melting point is $126-128^{\circ} \mathrm{C}$.

Nordihydro guaretic acid (NDGA)

Chemically defined as 2,3-dimetyl-1,4-bis (3,4 dihydroxy phenyl) butane. It is a white, crystalline solid. It is soluble in dilute alkali and slightly soluble in water. Melting point is $184-185^{\circ} \mathrm{C}$.
Propyl gallate (PG)

Chemical structure<smiles>CCCOC(=O)c1cc(O)c(O)c(O)c1</smiles>

Figure 7: Propyl gallate (synthetic water insoluble antioxidant).

Physicochemical properties

It is a white crystalline powder, sparingly soluble in water. Soluble in alcohols. Melting point is $146-148^{\circ} \mathrm{C}$.

Octyl gallate and dodecyl gallate

Chemical structures

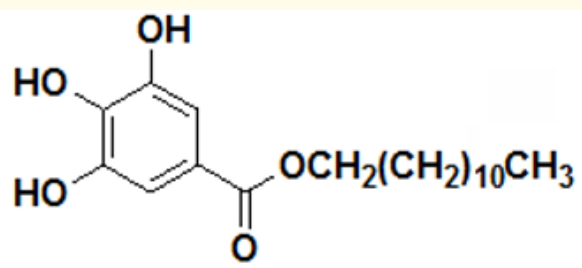

Figure 8: Dodecyl gallate (synthetic water insoluble antioxidant).

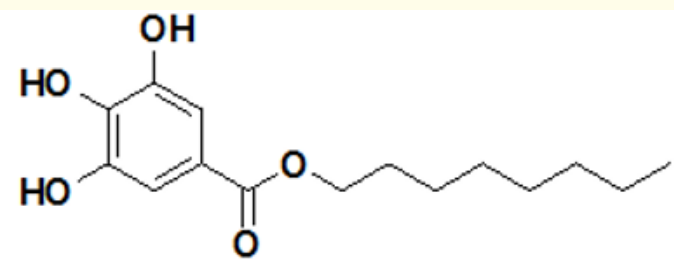

Figure 9: Octyl gallate (synthetic water insoluble antioxidant).

Physicochemical properties

Octyl gallate and dodecyl gallate are white to creamy crystalline powder, insoluble in water and have melting points of $91-92^{\circ} \mathrm{C}$.and $94-96^{\circ} \mathrm{C}$ respectively. 


\begin{tabular}{|c|c|c|}
\hline Antioxidant & Advantages & Disadvantages \\
\hline Synthetic & $\begin{array}{l}\text { Wide range of antioxidant activity } \\
\text { Common components of food are not } \\
\text { subject to any legislative restriction } \\
\text { Many chemical compounds can be found in human diet } \\
\text { High aqueous solubility } \\
\text { Pure, efficient, inexpensive } \\
\text { Easily available } \\
\text { Harmless if added in concentration permitted by legislation } \\
\text { Pharmacological effective against different diseases } \\
\text { Shorter duration of assay }\end{array}$ & $\begin{array}{c}\text { Low antioxidant efficiency } \\
\text { High usage level } \\
\text { Complex mixtures of many compounds of } \\
\text { different activities therefore subject to } \\
\text { physical/chemical interactions. } \\
\text { Expensive } \\
\text { Assay could be time-comsuming. } \\
\text { High degree of toxicity } \\
\text { Low aqueous solubility } \\
\text { Suspect of being chemicals } \\
\text { Subject to radiosensitization }\end{array}$ \\
\hline
\end{tabular}

Table 2: Advantages and Disadvantages of Natural and synthetic antioxidants.

Relationship between chemical structures and biological activities of antioxidant

Natural antioxidant tocopherol (vitamin E) shall be used to demonstrate relationship between chemical structures and antioxidant activity. Tocopherol interrupts free radical chain reactions by capturing the free radicals. The free hydroxyl group on the aromatic ring is responsible for the antioxidant properties. The chemical structures and biological activity are given in Figure 10 and Table 3 respectively.
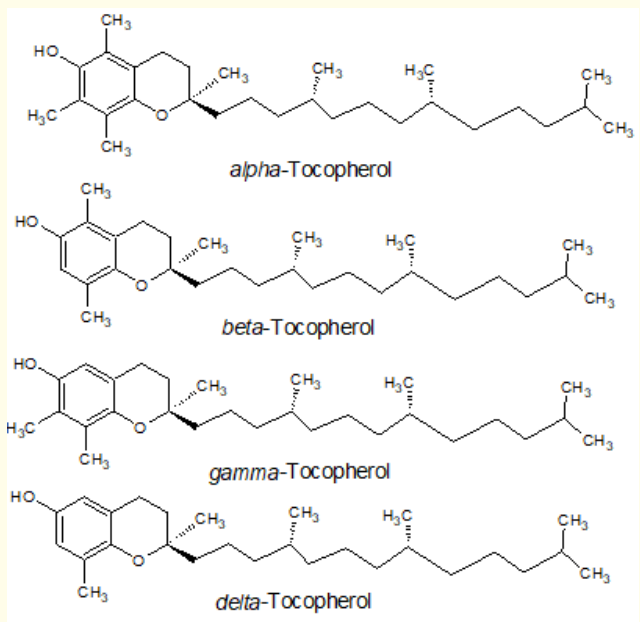

Figure 10: Chemical structures of tocopherol isomers (natural water insoluble vitamins).

\begin{tabular}{|l|c|}
\hline \multicolumn{1}{|c|}{ Antioxidant } & $\begin{array}{c}\text { Biological activity } \\
\text { (Int. unit/mg) }\end{array}$ \\
\hline d-alpha tocopherol & 1.49 \\
\hline d,l- alpha tocopherol & 1.10 \\
\hline d- gamma tocopherol & 0.15 \\
\hline d-delta tocopherol & 0.05 \\
\hline
\end{tabular}

Table 3: Structure activity relationship of tocopherol isomers.

Antioxidants in human body system

An extensive range of antioxidant defenses, both endogenous and exogenous, are present in human body system to protect cellular components from free radical induced damage. They are grouped into three main groups: antioxidant enzymes, chain breaking antioxidants, and transition metal binding proteins.

\section{Enzymatic antioxidants}

Catalase: catalyses the conversion of hydrogen peroxide to water and oxygen. It consists of four protein subunits, each containing a haem group and a molecule of NADPH [37]. The enzyme is largely located within cells in peroxisomes, which also contain most of the enzymes capable of generating hydrogen peroxide.

$$
\text { Catelase- } \mathrm{Fe}^{3+}+\mathrm{H}_{2} \mathrm{O}_{2} \longrightarrow \mathrm{O}_{2}+2 \mathrm{H}_{2} \mathrm{O}------- \text { Eq. } 22 \text {. }
$$


Superoxide dismutase (SOD) -catalyzes the detoxification of superoxide radical $\left(\mathrm{O}_{2}^{-\cdot}\right)$ to hydrogen peroxide $\left(\mathrm{H}_{2} \mathrm{O}_{2}\right)$ and oxygen. The hydrogen peroxide must then be removed by catalase or glutathione peroxidase as indicated in equation 22 . They are three forms of superoxide dismutase in mammalian tissues [38]. They include (a) Copper zinc superoxide dismutase (CuZnSOD) which is found in the cytoplasm and organelles of virtually all mammalian cells, (b) Manganese superoxide dismutase (MnSOD) which is found in the mitochondria of almost all cells, (c) extracellular superoxide dismutase (ECSOD) which is synthesized by only a few cell types, such as fibroblasts and endothelial cells. It is the major SOD detectable in extracellular fluids and is released into the circulation from the surface of vascular endothelium following heparin injection.

Glutathione peroxidase - catalyzes the oxidation of glutathione at the expense of a hydroperoxide, which might be hydrogen peroxide or another species such as a lipid hydroperoxide [39]. Glutathione peroxidase requires selenium or any of these essential minerals such as copper, manganese and zinc at the active site. The enzyme is widely distributed in almost all tissues, however, highest concentrations are found in liver. The activity of the enzyme is dependent on the constant availability of reduced glutathione.

\section{Chain breaking antioxidants (radical scavengers)}

These are compounds that stop chain propagation by blocking or reacting with free radicals generated in the initiation stage of oxidation. To block the radicals, the scavengers donate hydrogen atoms that react with alkyl or peroxy radicals, leading to the formation of quinones or quinine imines [40]. Such radical scavengers include lipid phase types (tocopherols, ubiquinol, carotenoids, flavonoids and aqueous phase type (ascorbic acid, uric acid, glutathione and other thiols).

\section{Transition metal binding proteins (metal deactivators)}

These are chelating agents that function by trapping metal ions in their structure in the form of stable complexes to reduce the catalytic oxidation activity of the metal ions. Many proteins bind copper and iron ions and this can make the protein a target of attack by hydroxide radical $(\mathrm{OH})$. Therefore, it is important for organisms that free transition metals in biological fluids are restricted to very low levels. Such chelating proteins are ferritin, lactoferrin and transferring [41].

\section{Application of antioxidants}

\section{Antioxidants as preservatives}

The addition of antioxidants to foods as preservatives, are usually done during various stages of production. Antioxidants are mostly added to fats and oils used in food production, other food products like: (i) vegetables and their products (ii) cereals and their bakery products, (iii) fruits and their products (iv) milk and their products (v) meat and their products, (vi) fish and their products, (vii) dried foods like sugars, honey, beverage. Antioxidants can also be added to packaging materials such as polyethylene plastics, paper boards to prevent oxidation of the materials or permitting the antioxidants to migrate into the foods and prevent the degradation of foods during processing and storage [42].

\section{Antioxidants in Prophylactic agents}

Antioxidants may function as immune modulators and are emerging as potential prophylactic agents in many diseases. Supplements of exogenous antioxidants can act directly to stop the free radical reactions, prevent lipid peroxidation, boost the endogenous antioxidant system and hence deliver the prophylactic activity. A number of antioxidants namely; $\beta$-carotene, coenzyme Q10, ellagic acid, epigallocatechin-3-0-gallate, genistein, lycopene, indole-3-carbinol, quercetin, vitamin $\mathrm{C}$ and vitamin $\mathrm{E}$ have been found to be pharmacologically active as prophylactic agents against cancer, cardiovascular diseases etc. Furthermore, cereals, legumes, spices, vegetables, fruits, crude palm oil, soybean oil, cod liver oil, sprouts, peppers, whole grain, honey, walnuts and black tea can prevent cardiovascular disease induced by oxidative stress, due to presence of unique dietary antioxidant components. Thus targeting oxidative stress or boosting the endogenous levels of antioxidants by the use of antioxidants is likely to have beneficial outcome in the management of several disorders [43].

Antioxidants as therapeutic agents [44-46]:

Cardiovascular Disease

Antioxidant vitamins by potentiating endothelial nitric oxide levels, increase in plasma antioxidant capacity, inhibit vascular inflammation, lipid peroxidation, platelet aggregation and oxidation of low density lipoproteins (LDL) can be beneficial in the treatment of endothelial dysfunction. Other potential mechanism of actions include reduction in intracellular ROS generation, induction of GSH, reduction in matrix metalloproteinase production, anti- inflammatory responses related to cardiovascular health. 


\section{Cancer}

Antioxidant diet or consumption of antioxidants like vitamins $\mathrm{E}, \mathrm{C}$, and selenium may have potential role in enhancing the efficacy of cancer treatment [40]. Vitamin E acts by increasing (a) humoral antibody production, (b) resistance to bacterial infections (c) cell-mediated immunity, (d) T lymphocyte response. It also inhibits mutagen formation, repairs membranes and DNA, blocks nitrosamine formation. Vitamin $\mathrm{C}$ displays its anti-carcinogenic activity by (a) blocking the formation of nitrosamines and fecal mutagens; (b) enhancing the immune response; (c) accelerating detoxification of liver enzymes.Antioxidants such as l-cysteine, $\mathrm{N}$-acetyl cysteine (NAC), thiols, green tea polyphenolscan block activation of NF- $\kappa B$ while $\beta$-carotene through its photoprotective property helps to protect against ultraviolet (UV)-light-induced cancer. Other antioxidants may act by (a) scavenging constitutive $\mathrm{H}_{2} \mathrm{O}_{2}$ including other free radicals or generate additional amounts of $\mathrm{H}_{2} \mathrm{O}_{2}$ to inhibit proliferation of cancer cells, (b) preserving normal cell cycle regulation, (c) inhibition of proliferation and inducing apoptosis, (d) inhibition of tumour invasion and angiogenesis (e) suppression of inflammation, and stimulation of phase II detoxification enzyme activity.

\section{Diabetes}

Antioxidant vitamins and phytochemicals with antioxidant activities are used to manage patients with diabetes and also prevent diabetic complications. Such phytochemicals include cinnamic acids, coumarins, diterpenes, flavonoids, lignans, monoterpenes, phenylpropanoids, tannins and triterpenes. They act by decreasing lipid peroxidation and oxidative stress markers in diabetic patients.

\section{Rheumatoid Arthritis}

Antioxidants like vitamins, $\beta$-cryptoxanthin, supplemental zinc, fruits and cruciferous vegetables are potential agents for therapeutic management of rheumatoid arthritis. Different studies have reported that the use of antioxidants as supplements with the conventional drugs yields even better results $n$ the treatment or rheumatoid arthritis. They act by increasing total thiols, glutathione resulting in the lowering of oxidative stress and the resultant inflammatory damage.

\section{Neurodegenerative diseases}

Dietary intake or supplementary intake of natural antioxidants like vitamin $C$, vitamin $E$, $\beta$-carotene can ameliorate a variety of neurological disorders. Also use of antioxidants like flavanoid, coenzyme Q10, $\alpha$-lipoate,melatonin, phenyl-alpha-tert butyl nitrone and GSH-glycosid have shown beneficial effect in neurodegenerative diseases. These antioxidants act as therapeutic barrier to oxidative stress by preventing oxidation of proteins, lipid peroxidations and generation of reactive oxygen species (ROS).

\section{Gastrointestinal diseases}

Antioxidant is most the effective drug in the treatment of chronic inflammatory bowel disorders. Natural antioxidants, like vitamins $\mathrm{A}, \mathrm{C}$ and $\mathrm{E}$ have been used in the treatment of gastrointestinal tract diseases. Synthetic antioxidants such as 5-amino-salicylic acid seems to be the such as $\mathrm{N}$-acetyl cysteine, biotin, selenium, zinc, manganese, copper, magnesium, folic acid and coenzyme $Q$ have also been used in GIT diseases such as liver disease and acute pancreatitis.

\section{Renal disorders}

Ascorbic acid (vitamin C) is most prominent antioxidants, exerting beneficial effects by an inhibition of lipidperoxidation and by reducing endothelial dysfunction. Other antioxidants employed in renal disorders are selenium and vitamin $\mathrm{E}$.

\section{Pulmonary disorders}

Administration of vitamin $\mathrm{C}$, vitamin $\mathrm{E}$, or combination of both, selenium, zinc, and garlic oil have shown beneficial effects in pulmonary disorders. VitaminE act by preventing the chain reaction involved in lipid peroxidation, while vitamin $\mathrm{C}$ acts to quench radicals inside the cell. Flavanoids as scavengers of nitric oxide have helped to reduce asthma inflammation by decreasing histamine release, arachidonic acid metabolism and cytokine productions [49]. Treatment of chronic obstructive pulmonary disease (COPD) has been achieved using thiol antioxidants and mucolytic agents namely glutathione,N-acetyl-L-cysteine, erdosteine, fudosteine, carbocysteine and dietary polyphenols (curcumin, resveratrol, catechins/quercetin. Furthermore, increase in selenium administration may suppress asthma inflammation by saturating glutathione peroxidase and down-regulates the transcription factor $\mathrm{NF}-\mathrm{Kb}$ 


\section{Eye disorders}

Antioxidants are beneficial in the treatment of eye disorders. Vitamin E, vitamin A and carotenoids like alpha-carotene, betacarotene, beta cryptoxanthin, lycopene, zeaxanthin and lutein confer beneficial effect in the management of cataract and other eye disorders.

\section{Infertility and pregnancy}

Antioxidant supplements have shown very significant beneficial effect in the treatment of infertility in male and female by assisting endogenous enzymatic antioxidants to nullify the oxidative stress. Endogenous and exogenous antioxidants such as glutathione, vitamin E, vitamin $\mathrm{C}$, N-acetylcysteine, taurine, hypotaurine and coenzymeQ10 increase sperm motility and prevent sperm mortality by reducing oxidative stress. Oral administration of coenzyme Q10 improved fertilization rate while administration of vitamin $\mathrm{C}$ along with vitamin $\mathrm{E}$ and glutathione through the same route showed significant reduction in hydroxyl glutathione levels in spermatozoa and also cause increase in sperm count.

Vitamins such vitamin $C$, vitamin $E$ and $\beta$-carotine etc. are found to be effective in the management of miscarriage and abortion. Trasferrin by suppressing ROS generation has been found effective for healthy follicle development.

\section{Aging}

The human body has several endogenous antioxidants to eliminate free radicals. Exogenous antioxidants have been effectively used to reduce free radical reactions since free radical damage to biological molecules has been implicated as the principal cause of aging. Antioxidants such as $\mathrm{N}$-acetylcysteine, butyl hydroxytoluene, vitamin C, vitamin E, polyphenols, carotenoids, coenzyme Q10 and ergothioneine have shown some life-extending effects. They do this by effectively enhancing resistance to oxidative stress and thus prevent/improve skin aging.

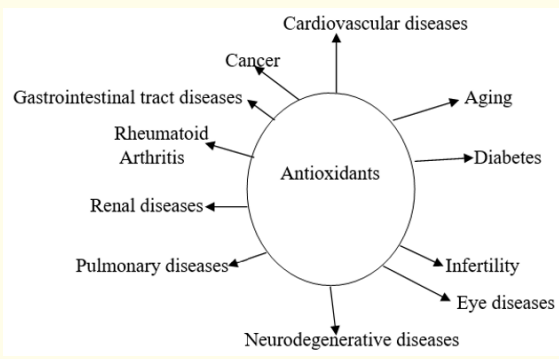

Figure 11: Role of oxidants (causing oxidative stress) and antioxidants to human diseases.
Pharmacokinetics of antioxidants

The pharmacokinetics of some major classes of antioxidants include [47-50]:

\section{Carotenoids}

Common food carotenoids present in fresh vegetables/salads and in fruits, (bananas, mango and papaya) have been found to be bioavailable. The beta-carotene has blood serum of 0.28-0.52 micro mol/L while blood serum of 0.2-0.28 and 0.29-0.60 have been obtained for lutein and lycopene respectively. All-trans-carotenoids have a better bioavailability than the 9-cis-forms. Absorption is thought to occur by passive diffusion following release from the food matrix in the gastrointestinal tract. The degree of bioavailability depends on the type and original concentration of the carotenoid (s). Food additives, such as oil or co-administration of soy germ or testosterone have enhanced the bioavailability of carotenoid compounds. Frequent meals and higher dietary contents have also enhanced the bioavailability of many carotenoids. Electrolytes such as calcium and magnesium ions have shown significant inhibition effect on the bioavailability of carotenoids. In general, the relative bioavailability of carotenoids varies from less than $10 \%$ in raw, uncooked vegetables to $50 \%$ in oils or commercial preparations. Dietary carotenoids following distribution could accumulate in many tissues including the liver, adipose, serum, breast milk, adrenal, prostate, macula, kidney, lung, brain, and skin. Elimination of carotenoids takes several days with elimination half-life of 5-7 and 2-3 days for beta-carotene and lycopene, respectively. Several oxidized metabolites of carotenoids are known.

\section{Polyphenols}

Polyphenols show poor bioavailability probably due to such factors as poor absorption, instability, excessive metabolism or intestinal microbial transformation.

Different dietary polyphenols show different rates of absorption and bioavailability. For example, isoflavones are the best absorbed dietary flavonoids, flavanols, flavanones and flavonol glycosides are intermediate, whereas proanthocyanidins, flavanol gallates and anthocyanins are the least absorbed. Absorption into systemic circulation which is by passive diffusion may be influenced by the matrix in which they are consumed. Absorption is also affected by dosage, vehicle of administration, antecedent diet, sex differences, individual genetic properties and the microbial population of the colon. Despite low bioavailability many polyphenols still display therapeutic antioxidant activities. 
Microemulsion, nanoemulsions and other nano-size formulations have been found to enhance the bioavailability of polyphenolic compounds. Distribution into tissues is rapid. Liver is the crucial organ responsible for various biotransformations of polyphenols. However, the intestinal mucosa, kidney and other tissues are also involved in the metabolism of polyphenols. The most abundant metabolic biotransformation of polyphenols are oxidation, reduction, hydrolysis and conjugation with 0-methylation, sulphate and glucuronate. Excretion is by bile recirculation or directly by the enterocyte back to small intestine reaching the colon with different chemical structures. Enhanced excretion in urine of easily recognizable conjugates, have been observed in foods with higher fat contents.

\section{Vitamins}

- Vitamin A - The beta-carotene has blood serum of 0.28-0.52 micro mol/L, 0.2-0.28 for lutein, and from 0.29-0.60 for lycopene. The bioconversion of beta-carotene to retinal is dose-dependent, and ranges between $27 \%$ and $2 \%$ for a 6 and $126 \mathrm{mg}$ dose, respectively. Several oxidized metabolites of carotenoids are known. Flavonols such as quercetin glycosides and rutin are predominantly absorbed as aglycones, bound to plasma proteins and subsequently conjugated to glucuronide, sulfate, and methyl moieties. The elimination half-life ranges from 12-19 hours.

- Vitamin C - is readily absorbed from the small intestines, peritoneum and subcutaneous tissues following dietary intake. It passes through the portal vein into general circulation. The bioavailability of vitamin $C$ is dose-dependent. Saturation of transport occurs with dosages of 200-400 mg/day. Adrenal cortex and medulla, pituitary, liver and corpus luteum store vitamin $\mathrm{C}$ up to an optimal saturation levels. It is distributed throughout the body and not protein-bound. The elimination half-life is about 10 hours. Excess Vitamin C is excreted in urine.

- Vitamin E ( $\alpha$-tocopherol) - is the most abundant tocopherol in human tissue. Its bioavailability is estimated to be $50 \%$. It has elimination half-life of 81 hours. As a fat-soluble vitamin, intestinal absorption, hepatic metabolism and cellular uptake of vitamin E follows that of other lipophilic molecules. The metabolism is primarily located in the liver. Conjugation of the metabolites takes place during metabolism, resulting predominantly in sulfated and glucuronidated metabolites. Glycine-glucuronide and taurine-modified metabolites of vitamin $\mathrm{E}$ have also been reported.

\begin{tabular}{|c|c|c|}
\hline $\begin{array}{c}\text { Natural } \\
\text { antioxidant }\end{array}$ & \multicolumn{2}{|c|}{ Recommended daily allowance } \\
\hline & Infants & Adults \\
\hline Vitamin A & $300-400 \mu \mathrm{g} /$ day & $700-900 \mu \mathrm{g} /$ day \\
\hline Vitamin C & $40-60 \mathrm{mg} /$ day & $60-80 \mathrm{mg} /$ day \\
\hline Vitamin E & $4-7 \mu \mathrm{g} / \mathrm{day}$ & $11-15 \mu \mathrm{g} /$ day \\
\hline Quercetin & & $100 \mathrm{mg} /$ single dose \\
\hline Epicatechin & & $82 \mathrm{mg} /$ single dose \\
\hline
\end{tabular}

Table 4: Therapeutic concentrations of some natural antioxidants.

\section{Conclusion}

Oxidative stress that causes tissue damage occurs in cells as a consequence of normal physiological processes and environmental interactions. Biological defense systems play vital roles in protecting body cells against oxidative damage. Oxidative damage leads to a variety of human diseases such cancer, neurodegenerative diseases, diabetes, rheumatoid arthritis, gastrointestinal disorders etc.

Natural and/or synthetic antioxidants have been used successfully as prophylactic or therapeutic agents for these human diseases. However better understanding of the biochemical events occurring at a cellular level to cause oxidative damage is required to direct future development of antioxidants with greater therapeutic effectiveness.

\section{Acknowledgement}

The authors would like to thank Dr, Akachukwu I, for preparing the chemical structures.

\section{Conflict of Interest}

There is no conflict of interest in the preparation of the manuscript.

\section{Bibliography}

1. Cutson TM., et al. "Pharmacological and non pharmacological interventions in the treatment of Parkinson disease". Physical Therapy 75 (1995): 363-373.

2. Jenner Pand Olanow CV. "Oxidative stress and the pathogenesis of Parkinson's disease”. Neurology 47 (1996): S161-S170.

3. Ahmadinejad F., et al. "Review molecular mechanisms behind free radical scavengers function against oxidative stress". Antioxidants 6 (2017): 51-66. 
4. Nose K. "Role of reactive oxygen species in the regulation of physiological functions". Biology and Pharmacy Bulletin 23 (2000): 897-903.

5. Valko M., et al. "Free radicals, metals and antioxidants in oxidative stress-induced cancer". Chemical and Biological Interaction 160 (2006): 1-40.

6. Sena LA and Chandel NS. "Physiological roles of mitochondrial reactive oxygen species”. Molecular Cell 48 (2012): 158-167.

7. Thannickal VJ and Fanburg BL. "Reactive oxygen species in cell signalling". American Journal of Physiology of Lung Cell and Molecular Physiology 279 (2000): L1005-L1028.

8. Hassan SZ., et al. "Oxidative stress in systemic lupus erythematosus and rheumatoid arthritis patients: Relationship to disease manifestations and activity". International Journal of Rheumatoid Disease 14 (2011): 325-331.

9. Altieri F., et al. "DNA damage and repair: From molecular mechanisms to health implications". Antioxidative and Redox Signalling 10 (2008): 891-938.

10. Banerjee B., et al. "Biochemical effect ts of some pesticides on lipid peroxidation and free-radical scavengers". Toxicology Letters 107 (1999): 33-40.

11. Jomova K and Valko M. "Advances in metal-induced oxidative stress and human disease". Toxicology 283 (2011): 65-87.

12. Rhee SG. "H2O2 a necessary evil for cell signalling". Science 312 (2006): 1882-1883.

13. Decoursey $\mathrm{T}$ and Ligeti E. "Regulation and termination of NADPH oxidase activity". Cellular Molecular Life Science 62 (2005): 2173-2193.

14. Vergely C. "Nitric oxide synthase inhibition and oxidative stress in cardiovascular diseases: Possible therapeutic targets?" Pharmacology and Therapeutics 140 (2013): 239-257.

15. Maritim A C., etal. "Diabetes, oxidative stress, and antioxidants: A review". Journal of Biochemistry and Molecular Toxicology 17 (2003): 24-38.
16. Esposito E., et al. "A review of specific dietary antioxidants and the effects on biochemical mechanisms related to neurodegenerative processes". Neurobiology and Aging 23 (2002): 719-735.

17. Scherman D., et al. "Striatal dopamine deficiency in Parkinson's disease role of aging". Annal of Neurology 26 (1989): 551-557.

18. Feig DI., et al. "Reactive oxygen species in tumorigenesis". Cancer Research 54.7 (1994): 1890-1894.

19. Haigh RC., et al. "Myeloperoxidase and oxidative stress in rheumatoid arthritis". Rheumatology 51 (2012): 1796-1803.

20. Fang YZ., et al. "Free radicals, antioxidants and nutrition". Nutrition 18 (2002): 872-879.

21. Luciak M. "Oxidative stress and human diseases". Annales Academiae Medicine Bialostocensis 49 (2001): 157-161.

22. Dozor A.J. "The role of oxidative stress in the pathogenesis and treatment of asthma". Annals of the New York Academy of Sciences 1203 (2010): 133-137.

23. Guilliams TG. "Free radicals, antioxidants and eye diseases". The Standard 2 (1999): 1-8.

24. Goldenberg HA. "Regulation of mammalian iron metabolism: current state and need for further knowledge". Critical Review of Clinical Laboratory Sciences 34 (1997): 529-572.

25. Aruoma OI and Halliwell B. "Superoxide-dependent and ascorbate-dependent formation of hydroxyl radicals from hydrogen peroxide in the presence of iron". Biochemistry Journal 241 (1987): 273-278.

26. Finkel T. "Oxidants, oxidative stress and the biology of aging". Nature 408 (2000): 239-248.

27. Halliwell B. "How to characterize biological antioxidants". Free Radical Research Communication 9 (1990): 1-32.

28. Frankel EN and Meyer AS. "The problems of using onedimensional methods to evaluate multifunctional food and biological antioxidants". Journal of Science Food and Agriculture 80 (2000): 1925-1941. 
29. Chipault JR. "Antioxidants for use in food. In Autooxidation and Antioxidants" Interscience 2 (1962): 477-542.

30. Valko M., et al. "Free radicals and antioxidants in normal physiological functions and human disease" International Journal of Biochemistry and Cell Biology 39.1 (2007): 44-84.

31. Gupta VK and Sharma SK. "Plants as natural antioxidants". Natural Product Radiance 5.4 (2006): 326-334.

32. Hurrell R. "Influence of vegetable protein sources on trace element and mineral bioavailability". Journal of Nutrition 133.9 (2003): 2973S-2977S.

33. Alok SH., et al. "Herbal antioxidant in clinical practice (): A review". Asian Pacific Journal of Tropical Biomedicine 4.1 (2014): 78-84.

34. Cieślik E., et al. "Content of polyphenols in fruit and vegetables". Food Chemistry 94 (2006): 135-142.

35. Li Y. "Antioxidant in biology and medicine: essentials, advances, and clinical applications". Nova Science Publishers USA (2011): 22.

36. Andre C., et al. "Analytical strategies to evaluate antioxidants in food: a review". Trends in Food Science and Technology 21 (2010): 229-246.

37. Kirkman HN., et al. "The function of catalase-bound NADPH". Journal of Biological Chemistry 262 (1987): 660-665.

38. Weisiger RA and Fridovich I. "Mitochondrial superoxide dismutase: site of synthesis and intra mitochondrial location". Journal of Biological Chemistry 248 (1973): 4793-4896.

39. Brigelius-Flohe R. "Tissue-specific functions of individual glutathione peroxidases". Free Radical Biological Medicine 27 (1999): 951- 965.

40. Nishiyama T., et al. "Chain-breaking fused heterocyclic antioxidants: Antioxidant activities of phenothiazines compared to related compounds".Polymer Degradation and Stability 64 (1999): 33-38.
41. Zadák Z., et al. "Antioxidants and vitamins in clinical conditions". Physiology Research 58.1 (2009): S13-S17.

42. Morton LW., et al. "Chemistry and biological effects of dietary phenolic compounds: Relevance to cardiovascular disease". Clinical Experimental Phamarcology and Physiology 27 (2000): 152-159.

43. Ratnam DV., et al. "Role of Antioxidants in Prophylaxis and Therapy: A Pharmaceutical Perspective". Journal of Controlled Release 113.3 (2006): 189-207.

44. Noguchi N. "Novel insights into the molecular mechanisms of the antiatherosclerotic properties of antioxidants: The alternative to radical scavenging". Free Radical of Biological Medicine 33 (2002): 1480- 1489

45. Lades EJ., et al. "Antioxidants and cancer therapy". Journal of Clinical Oncology 22.3 (2004): 517-528.

46. Khan I., et al. "Antioxidant supplements and gastrointestinal diseases: A critical appraisal". Medical Principles and Practice 26 (2017): 201-217.

47. Parker RS., et al. "Bioavailability of carotenoids in human subjects". Proceedings Nutrition Society 58 (1999): 155-162.

48. Schwedhelm E., et al. "Clinical pharmacokinetics of antioxidants and their impact on systemic oxidative stress". Clinical Pharmacokinetics 42.5 (2003): 437-59.

49. Heim KE., et al. "Flavonoid antioxidants: Chemistry, metabolism and structure-activity relationships". Journal of Nutritional Biochemistry 13 (2002): 572-584.

50. Rigotti A. "Absorption, transport, and tissue delivery of vitamin E". Molecular Aspect and Medine 28 (2007): 423-436.

\section{Volume 3 Issue 6 June 2019}

(C) All rights are reserved by Ndiamaka H Okorie., et al. 